\title{
UNA NUEVA ESPECIE DE OXYPETALUM (APOCYNACEAE: ASCLEPIADOIDEAE) DE SANTA CATARINA, BRASIL
}

\author{
HÉCTOR A. KELLER ${ }^{1}$ \& LUIS A. FUNEZ ${ }^{2}$
}

\begin{abstract}
Summary: Keller, H. A. \& L. A. Funez. 2017. A new species of Oxypetalum (Apocynaceae: Asclepiadoideae) from Santa Catarina, Brazil. Bonplandia 26(2): 119-124.

Based on herbarium material collected at 1100 m.a.s.l. in the Santa Catarina State, Brazil, a new species of Oxypetalum R. Br. (Apocynaceae) is here described and illustrated. Its conservation status is assessed and information on its ecology are provided. Similarities and differences with related species are discussed.
\end{abstract}

Key words: Endangered plants, Oxypetalum marianae, taxonomic novelties.

Resumen: Keller, H. A. \& L. A. Funez. 2017. Una nueva especie de Oxypetalum (Apocynaceae: Asclepiadoideae) de Santa Catarina, Brasil. Bonplandia 26(2): 119-124.

Sobre la base de un ejemplar de herbario recolectado a 1100 m.s.n.m., en el estado de Santa Catarina, Brasil, se describe e ilustra una nueva especie del género Oxypetalum R. $\mathrm{Br}$. (Apocynaceae). Se provee información sobre su estado de conservación y se brindan observaciones ecológicas. Se discuten las similitudes y diferencias con especies afines.

Palabras clave: Novedad taxonómica, Oxypetalum marianae, plantas en peligro.

\section{Introducción}

Oxypetalum R. Br. (Apocynaceae, Asclepiadoideae) cuenta con cerca de 130 especies distribuidas desde el centro de la Argentina hasta México, pero con mayor concentración de diversidad en la Argentina, Brasil y Paraguay (Ezcurra et al., 2008; Farinaccio \& Mello-Silva 2006; Fontella Pereira et al., 2004). Se trata de un género creciente en número de especies debido a recientes hallazgos (Farinaccio \& Goyder, 2016; Farinaccio \& Keller, 2014; Farinaccio \&
Mello-Silva, 2006; Keller, 2015; Martín et al., 2017; Rapini \& Farinaccio, 2010) y también a nuevas combinaciones que han posibilitado la incorporación de representantes de otros géneros como Amblystigma Benth., Melinia Decne., Rhyssostelma Decne., Schistogyne Hook. \& Arn. y Widgrenia Malme (Liede \& Meve, 2015; Rapini et al., 2011).

Para la flora de Santa Catarina, Fontella Pereira et al. (2004) mencionaron un total de 21 especies, una de las cuales, Oxypetalum insigne (Decne.) Malme, cuenta con variedades que se caracterizan por presentar el apéndice

\footnotetext{
${ }^{1}$ Instituto de Botánica del Nordeste, CC 209, 3400-Corrientes, Argentina. Investigador CONICET. Facultad de Ciencias Forestales, Universidad Nacional de Misiones. E-mail: ibone@agr.unne.edu.ar

${ }^{2}$ Universidade Regional de Blumenau, Campus I, Herbário Dr. Roberto Miguel Klein (FURB), 89012-900, Blumenau, SC, Brazil.
} 
estigmático con ramas aplanadas, foliáceas. Una segunda especie con esta característica, O. hoehnei Malme (=Calostigma dusenii Hoehne) del estado de Paraná, es incluida en el tratamiento como de presencia probable para la flora catarinense, ocurrencia que ya había sido efectivamente certificada por un ejemplar recolectado en el año 1992 en la Sierra de Iquererim, Campo Alegre (Cordeiro \& Poliquesi 955 MBM, CTES). En esta contribución se describe e ilustra una nueva especie de Oxypetalum hallada en Santa Catarina. Al presentar este tipo de apéndice estigmático se emplea la especie más afín de estas dos como base comparativa para la diagnosis.

\section{Materiales y Métodos}

Con el fin de corroborar la novedad taxonómica se elaboró un listado actualizado de las especies del Cono Sur de América del Sur que han sido documentadas para el estado de Santa Catarina y regiones limítrofes (www.darwin.edu.ar). Posteriormente, para descartar la posibilidad de que se trate de una especie con distribución disyunta se amplió el listado revisando catálogos de amplio alcance geográfico en archivos impresos (Ezcurra, 1999; Ezcurra et al., 2008; Forzza, 2010) y digitales (www.tropicos.org; reflora.jbrj. gov.br). También se revisaron monografías regionales sobre el género clásicas y actuales (Farinaccio, 2004; Farinaccio \& Goyder, 2016; Fontella Pereira et al., 2010; Hoehne, 1916; Marquete et al., 2007; Meyer, 1943; Meyer \& Bacigalupo, 1979; Schulz, 1937) y contribuciones sobre nuevos taxa recientemente publicados (Farinaccio \& Keller, 2014; Martín et al., 2017; Farinaccio \& Mello Silva, 2006; Rapini, 2002; Rapini \& Farinaccio, 2010).

Una cámara fotográfica con alta resolución y un microscopio estereoscópico fueron utilizados para obtener imágenes empleadas en el proceso de estudio del material y para confeccionar las ilustraciones. El indumento fue examinado además con microscopio óptico. En el herbario CTES se estudiaron muestras de las dos especies afines.

\section{Resultados}

Oxypetalum marianae H. A. Keller \& Funez, sp. nov.

Figs. 1(A-G) y 2

Differt a O. hoehnei Malme foliis angustioribus, coronae lobulis fauci inclusis, late ovatis non appendiculatis, caudiculae horizontales.

Typus: BRASIL. Santa Catarina: Grão Pará, Serra do Corvo Branco, 28 03'19'S, 49 22'03'W, 1100 m, 01-X-2014, L. A. Funez 3457 (holotypus FURB 44967!, clastotypus CTES!).

Sufrútice latescente 0,80-1,50 m alt., ramificado en la base, ramas volubles, cilíndricas, glabrescentes en la base, hirsutas en el ápice, pelos multicelulares uniseriados, ca. 0,5 mm long., curvados, retrorsos y de superficie punteadorugosa; entrenudos 1,2-5,5 cm long. Hojas opuestas con pubescencia similar a la de los tallos; pecíolos 0,8-15 $\mathrm{mm}$ long., 0,6-0,8 $\mathrm{mm}$ diám.; láminas lanceoladas a estrechamente lanceoladas, a veces algo falcadas, 1,5-4,9 × 0,4-1,6 cm, discoloras, hirsutas, tricomas patentes; margen entero, ciliado, base profundamente cordada, con 2-4 coléteres cónicos en el haz, 0,2-0,4 mm long., ápice agudo, venación broquidódroma. Flores solitarias o en inflorescencias subaxilares racemosas, erectas, alternas, con 2-4 flores; pedúnculos 3-12 mm long., hirsutos; brácteas oblongo-lanceoladas, hirsutas, 1,5-2 × 0,25-0,3 $\mathrm{mm}$; pedicelos 5,5-10 mm long., pubescentes. Cáliz dividido hasta cerca de la base, verdeamarillento; tubo 0,6-0,8 $\mathrm{mm}$ long., lóbulos estrechamente lanceolados, agudos, 2,5-3 × 0,2$0,3 \mathrm{~mm}$, acuminados, dorsalmente pubescentes, ciliados, tricomas aciculares 0,1-0,35 mm long., con algunos tricomas cortos provistos de célula apical lanceolada de mayor diámetro que las demás células. Corola con el tubo campanulado 3-4 mm long., lóbulos lanceolados 2,5-3 × 0,2-0,3 mm, verde-amarillentos, a veces con máculas rojizas, reflexos y retorcidos en el tercio distal, cara abaxial con tricomas aciculares en toda su superficie, los más largos de ca. 0,4 mm se agrupan cerca del ápice, borde ciliado con tricomas aciculares y tricomas con célula apical mayor, cara adaxial papilosa y con pelos cortos de ca. 0,04 mm long. Corona blanca, carnosa ca. $1 \mathrm{~mm}$ alt.; lóbulos basalmente soldados, con 


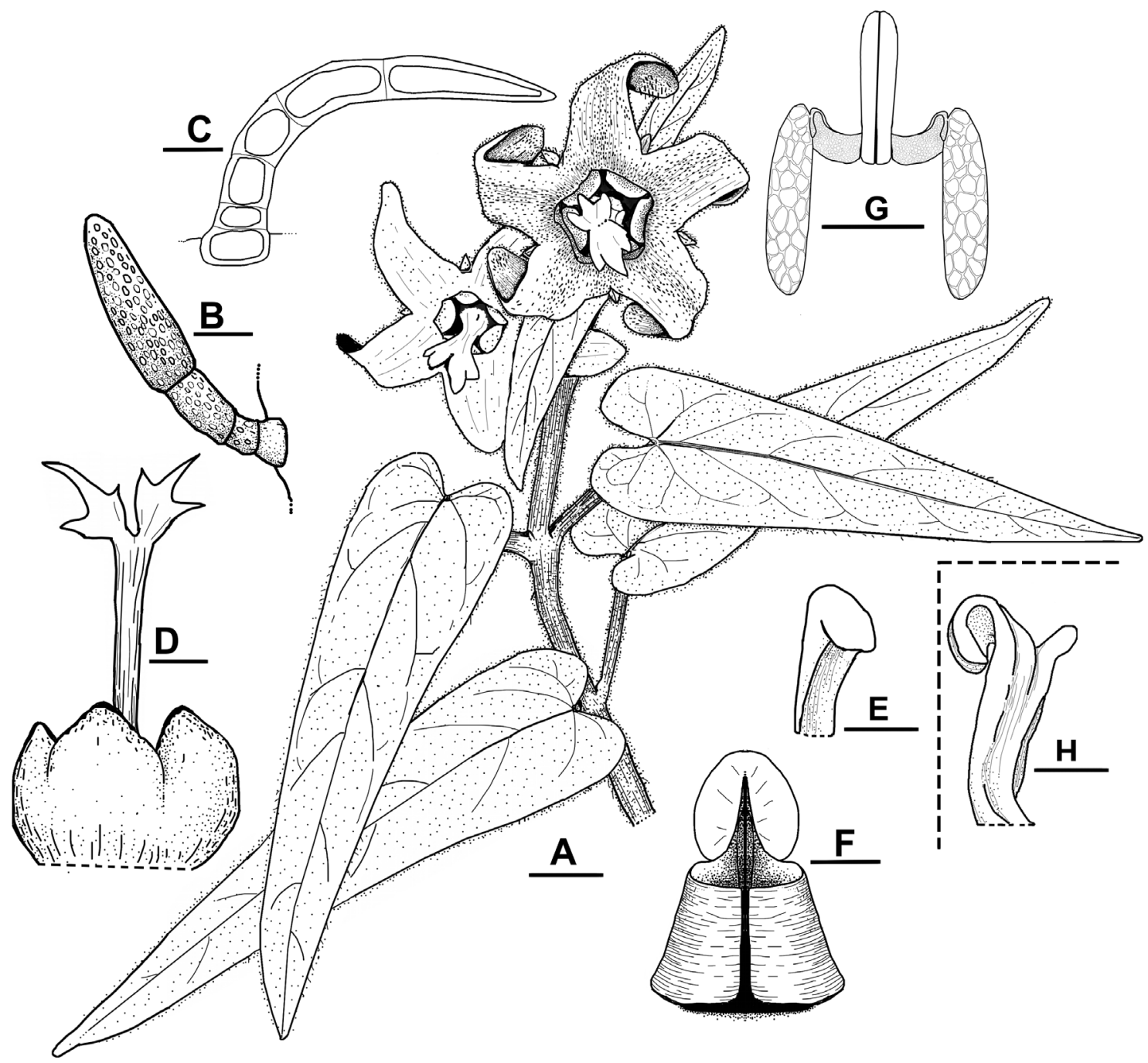

Fig. 1. Oxypetalum marianae H. A. Keller \& Funez. A: Rama con flores. B: Tricoma del borde de los lóbulos del cáliz, con célula apical mayor. C: Tricoma acicular de la cara abaxial de los lóbulos de la corola. D: Corona y apéndice estilar. E: Lóbulo de la corona en vista lateral. F: Antera en vista ventral. G: Polinario. (A-G: Funez 3457, holotipo FURB). Oxypetalum hoehnei Malme. H: Lóbulo de la corona en vista lateral (H: de Silva et al. 3248, CTES). Escalas en mm. A: 1,2; B: 0,05; C: 0,04; D: 0,5; E: 0,7; F: 0,25; G: 0,18; H: 0,7. Dibujos de H. A. Keller.

Fig. 1. Oxypetalum marianae H. A. Keller \& Funez. A: Branch with flowers. B: Trichome in the edge of calyx lobes, with greater apical cell. C: Acicular trichome in the adaxial face of the corolla lobes. D: Corone and stylar appendix. E: Corone lobe in side view. F: Anther in ventral view. G: Pollinarium. (A-G: Funez 3457, holotype FURB). Oxypetalum hoehnei Malme. H: Corone lobe in side view (H: de Silva et al., 3248, CTES) Scales in mm A: 1,2; B: 0,05; C: 0,4; D: 0,5; : 0,7; F: 0,25; G: 0,18; H: 0,7. Drawings by H. A. Keller.

la porción libre anchamente ovado-triangular $0,5-0,65 \times 0,7-0,9 \mathrm{~mm}$, incluida en el tubo de la corola, cara ventral con espesamiento distal triangular, resultante de pliegue apical soldado. Anteras de 0,9-1 × 0,6-0,7 mm, membrana apical ovada, $0,35-0,45 \times 0,3-0,4 \mathrm{~mm}$. Retináculo oblongo, algo más ancho en la parte distal, 0,25$0,3 \times 0,04-0,06 \mathrm{~mm}$; caudículas horizontales rectangulares ca. $0,13 \times 0,05 \mathrm{~mm}$, aplanadas, reticulado-translúcidas, sin espesamientos obscuros, dientes ni articulaciones; polinias oblongo-elípticas, $0,27-0,32 \times 0,04-0,05 \mathrm{~mm}$. Ginostegio blanco con tonos rosados, ca. $1 \mathrm{~mm}$ long, sésil, apéndice estigmático 4-4,2 mm long., blanco, exserto, bífido, ramas trífidas divergentes, aplanadas, foliáceas. Frutos desconocidos. 

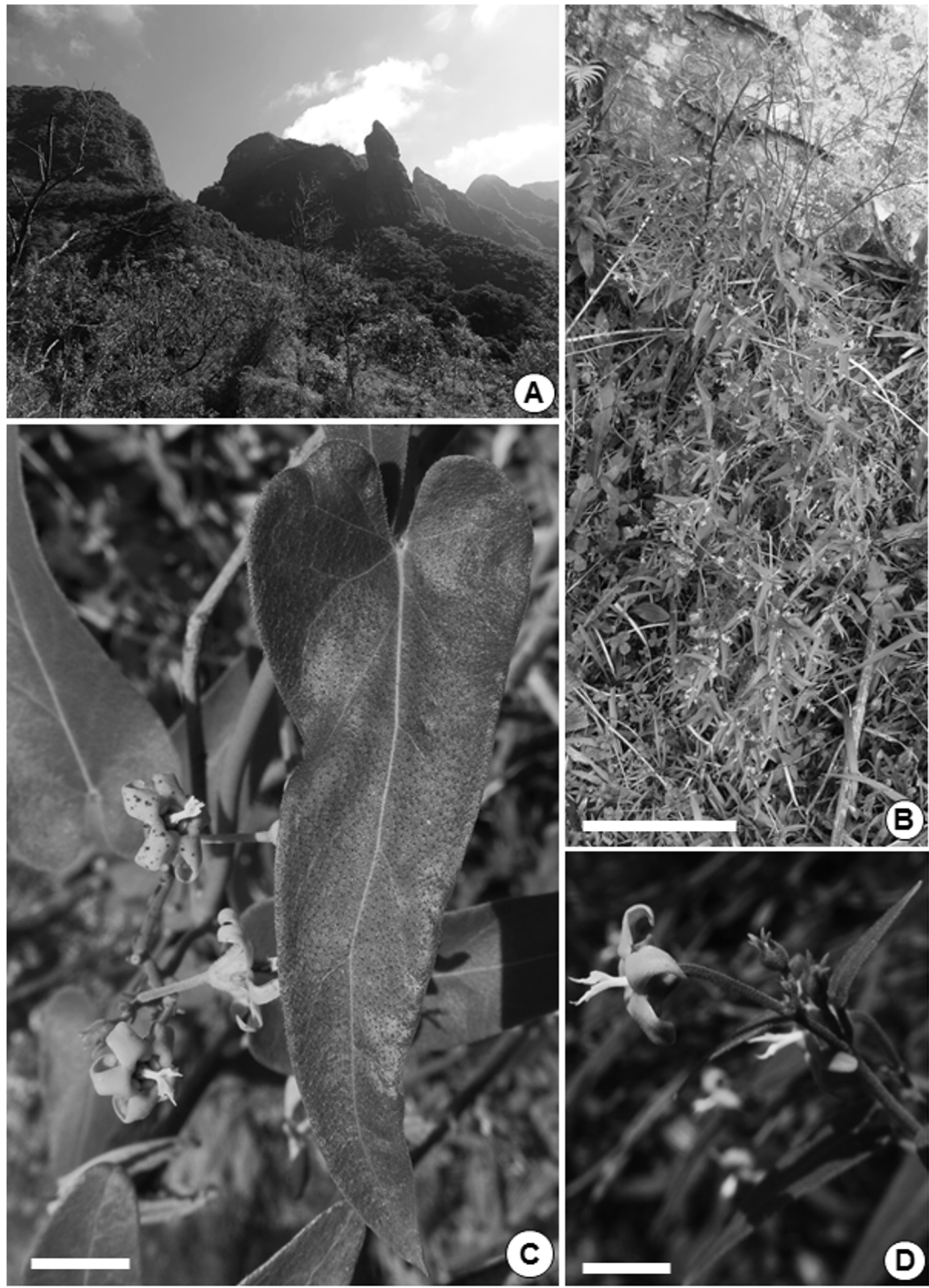

Fig. 2. A: Serra do Corvo Branco, localidad típica. B: Planta en su ambiente. C: Hoja y flores. D: Flor en vista lateral. (B-D: Funez 3457, holotipo FURB). Escalas en cm. B: 15; C: 0,4; C: 0,3 (Fotografías A: Keller; B-D: Funez).

Fig. 2. A: Serra do Corvo Branco, typical locality. B: Plant in its environment. C: Leaf and flowers. D: Flower in side view. (B-D: Funez 3457, holotype FURB). Scales in cm. B: 15; C: 0.4; C: 0.3 (Photographs A: Keller, B-D: Funez). 
Etimología: Dedicamos la especie a la Dra. Maria Ana Farinaccio del Herbário da Universidade Federal de Mato Grosso do Sul, Campus do Pantanal, especialista en taxonomía y filogenia del género Oxypetalum.

Distribución geográfica y hábitat: Conocida apenas por el holotipo, su distribución hasta el presente se restringe a la localidad de Serra do Corvo Branco, entre los municipios de Urubici e Grão Pará. Esta formación geológica forma parte de los Aparados da Serra Geral, región situada entre los estados de Rio Grande do Sul y Santa Catarina, donde existe un desnivel abrupto que alcanza los 700 metros de diferencia vertical entre el planalto y la planicie litoraleña, con la formación de muchos paredones y cañones. La especie habita áreas de vegetación herbáceoarbustiva entre afloramientos rocosos. La región suele presentar temperaturas bajo cero durante el invierno, con heladas y neviscas frecuentes, aunque con extrema humedad debido a la constante condensación de masas húmedas, las que provenientes del océano se encuentran con los paredones rocosos. Esta región es considerada como la que detenta el mayor número de endemismos en lo que respecta a plantas vasculares para el estado de Santa Catarina, tal como lo señalan Hassemer et al. (2015).

Conservación: Se trata de una especie conocida apenas por un ejemplar, en una región repleta de endemismos, sin protección legal y sujeta a proyectos de generación de energía eólica que se establecerán a escasa distancia de la localidad típica. Ante esta situación, se sugiere considerar a la especie en peligro crítico (CR), de acuerdo con los criterios de IUCN (2001).

Observaciones taxonómicas: De las dos especies que presentan apéndices estilares con ramas aplanadas foliáceas citadas para Santa Catarina, Oxypetalum insigne, es la que con mayor facilidad se distingue de $O$. marianae, ya que sus flores son de diámetro mucho mayor debido a los largos lóbulos de la corola; sus caudículas presentan dientes notables y su retináculo es mucho más largo que las polinias, caracteres que no presentan las otras dos especies. En O. hoehnei las caudículas son levemente descendentes y presentan un espesamiento obscuro sinuoso, mientras que en $O$. marianae las caudículas son horizontales, planas y enteramente traslúcidas. Los lóbulos de la corona son anchamente ovadotriangulares en $O$. marianae, y se encuentran ocultos en la garganta de la corola, mientras que en $O$. insigne y $O$. hoehnei son alargados, algo más anchos en la parte apical y, a la antesis, sobresalen por encima de la garganta. El espesamiento distal de dichos lóbulos (pliegue apical), ocurre en las tres especies, pero sobre dicho espesamiento se proyecta un apículo en $O$. insigne, al igual que en $O$. hoehnei (fig. $1 \mathrm{H}$ ); mientras que $O$. marianae, no presenta ningún tipo de apéndice (Fig. 1E). Vegetativamente $O$. marianae se distingue de estas dos especies por sus hojas pequeñas, estrechamente lanceoladas y profundamente cordadas.

\section{Bibliografía}

EZCURRA, C. 1999. Asclepiadaceae. En ZULOAGA, F, O. \& O. MORRONE (eds.), Catálogo de las Plantas Vasculares de Argentina II. Dycotiledoneae. Monogr. Syst. Bot. Missouri Bot. Gard. 74: 78-98.

EZCURRA, C., J. FONTELLA PEREIRA \& L. KINOSHITA. 2008. Apocynaceae (incl. Asclepiadaceae). En ZULOAGA, F. O., O. MORRONE \& M. BELGRANO (eds.), Catálogo de las Plantas Vasculares del Cono Sur, vol. 2. Monogr. Syst. Missouri Bot. Gard. 107: 1090-1143.

FARINACCIO, M. A. 2004. Oxypetalum R. Br. En WANDERLEY, M. G. L., G. L. SHEPHERD, T. S. MELHEM \& A. M. GIULIETTI (eds.) Flora fanerogâmica do Estado de São Paulo 4, pp. 130-150, Fapesp/RiMa, São Paulo.

FARINACCIO, M. A. \& R. MELLO-SILVA. 2006. Oxypetalum gyrophyllum and O. oblanceolatum, new species of Asclepiadoideae (Apocynaceae) from Brazil, and a key for the $O$. insigne group. Novon 16: 235-239.

FARINACCIO, M. A. \& H. A. KELLER. 2014. Novelties in Oxypetalum (Apocynaceae-Asclepiadoideae) for the Argentine flora. Phytotaxa 184: 109-114.

FARINACCIO, M. A. \& D. GOYDER. 2016. A synopsis of Oxypetalum (Apocynaceae) in Bolivia, with the description of one new species and a key to species in Bolivia. Phytotaxa 267: 17-28.

FONTELLA PEREIRA, J., M. C. VALENTE, N. M. S. MARQUETE \& C. L. F ICHASO. 2004. Apocináceas, Asclepiadaóideas. En REIS, A. (ed.) Flora Ilustrada Catarinense. Herbário Barbosa Rodrigues, Itajaí, 1-250 pp. 
FONTELLA PEREIRA, J., S. A. CÁCERES MORAL \& M. B. de GOES. 2010. Sinopsis y tipificaciones de las especies del género Oxypetalum R. Br. (Asclepiadaceae) en Paraguay. Notula ad Floram paraquaiensem. Candollea 65: 106-395.

FORZZA, R. C. 2010. Catálogo das Plantas e Fungos do Brasil, vol. 1. Andrea Jakobsson Estúdio and Rio de Janeiro Botanical Garden, pp. 619-644.

HASSEMER, G., P. M.A. FERREIRA \& R. TREVISAN. 2015. A review of vascular plant endemisms in Santa Catarina, southern Brazil, highlights critical knowledge gaps and urgent need of conservation efforts. J. Torrey Bot. Soc. 142: 78-95.

HOEHNE, F. C. 1916. Monografia das Asclepiadaceas brasileiras (Monographia Asclepiadacearum Brasiliensium). Commissão de Linhas Telegraphicas Estrategicas de Matto-Grosso ao Amazonas, Rio de Janeiro. Publicação 38, 191 pp.

IUCN. 2001. The IUCN Red List of Threatened Species, version 2001.4. IUCN Red List Unit, Cambridge, UK. Disponible http://www.iucnredlist. org/.

KELLER, H. A. 2015. Oxypetalum radinsii (Apocynaceae: Asclepiadoideae), una nueva especie endémica de Misiones, Argentina. Bonplandia 24: 51-56.

LIEDE, S. \& U. MEVE. 2015. Synonymy of three South American genera in Apocynaceae, and new combinations in Oxypetalum and Tassadia. Phytotaxa 202: 035-044.
MARQUETE, N. F. S., J. FONTELLA PEREIRA \& M. C. VALENTE. 2007. Asclepiadoideae (Apocynaceae) from southeastern Brazil. I. The genus Oxypetalum from Rio de Janeiro State, Brazil. Ann. Mo. Bot. Gard. 94: 435-462.

MARTÍN, C., C. A. ZANOTTI \& C. EZCURRA. 2017. A New Species of Oxypetalum (Apocynaceae, Asclepiadoideae) from Northern Argentina. Syst. Bot. 42: 578-583.

MEYER, T. 1943. Revisión de las especies argentinas del género Oxypetalum (Asclepiadaceae). Lilloa 9: 5-72.

MEYER, T. \& N. M. BACIGALUPO. 1979. Asclepiadaceae. En BURKART, A. (ed.), F1. II. Entre Ríos, Colecc. Ci. Inst. Nac. Tecnol. Agropecu. 6(5a): 103-147.

RAPINI, A. 2002. Oxypetalum rusticum (Apocynaceae, Asclepiadoideae), a new species from the Espinhaço Range, Minas Gerais, Brazil. Novon 12: 385-387.

RAPINI, A., \& M. A. FARINACCIO. 2010. Oxypetalum laciniatum, uma espécie nova de Asclepiadoideae (Apocynaceae) do sul da Bahia, Brasil. Rodriguésia 61: 017-020.

RAPINI, A., J. FONTELLA PEREIRA \& D. J. GOYDER. 2011. Towards a stable generic circumscription in Oxypetalinae (Apocynaceae). Phytotaxa 26: 9-16.

SCHULZ, A. G. 1937. Las asclepiadáceas del territorio del Chaco. Lilloa 1: 347-391.

Original recibido el 11 de septiembre de 2017; aceptado el 20 de octubre de 2017. 